\title{
Impact of neutral boundary-layer turbulence on wind-turbine wakes: A numerical modelling study
}

\author{
Antonia Englberger · Andreas Dörnbrack
}

5 Received: DD Month YEAR / Accepted: DD Month YEAR

6 Abstract The wake characteristics of a wind turbine in a turbulent boundary layer under neutral stratification are investigated systematically by means of large-eddy simulations. A methodology to maintain the turbulence of the background flow for simulations with open horizontal boundaries, without the necessity of the permanent import of turbulence data from a precursor simulation, was implemented in the geophysical flow solver EULAG. These requirements are fulfilled by applying the spectral energy distribution of a neutral boundary layer in the wind-turbine simulations. A detailed analysis of the wake response towards different turbulence levels of the background flow results in a more rapid recovery of the wake for a higher level of turbulence. A modified version of the Rankine-Froude actuator disc model and the blade element momentum method are tested as wind-turbine parametrizations resulting in a strong dependence of the near-wake wind field on the parametrization, whereas the far-wake flow is fairly insensitive to it. The wake characteristics are influenced by the two considered airfoils in the blade element momentum method up to a streamwise distance of $14 D$ ( $D=$ rotor diameter). In addition, the swirl induced by the rotation has an impact on the velocity field of the wind turbine even in the far wake. Further, a wake response study reveals a considerable effect of different subgrid-scale closure models on the streamwise turbulent intensity.

Keywords Atmospheric boundary layer · Large-eddy simulation · Turbulence - Wind-turbine wake

A. Englberger

Institut für Physik der Atmosphäre, DLR Oberpfaffenhofen

E-mail: antonia.englberger@dlr.de

A. Dörnbrack

Institut für Physik der Atmosphäre, DLR Oberpfaffenhofen

E-mail: andreas.doernbrack@dlr.de 


\section{Introduction}

Wind turbines operate in the atmospheric boundary layer (ABL) where atmospheric turbulence arises from velocity shear (velocity change with height) and directional shear (wind direction change with height), thermal stratification, low-level moisture, as well as from the interaction of the airflow with vegetation, buildings or terrain (Naughton et al., 2011; Emeis, 2013, 2014). ABL turbulence affects the velocity deficit and the turbulence in the wake, having a large impact on energy production, on fatigue loading, and on the life expectancy of wind turbines. Numerical simulations of wind turbines in the ABL have become an important tool in the investigation of these complex processes. Different numerical approaches exist to simulate the impact of ABL turbulence on wind-turbine wakes. Here, we focus on a large-eddy simulation (LES), being an approved tool to study the turbulence in the ABL (Bellon and Stevens, 2012).

The influence of a turbulent flow on the structure of the wake has been investigated in experimental studies (Medici and Alfredsson, 2006; Chamorro and Porté-Agel, 2009; Zhang et al., 2012) as well as in numerical simulations (Troldborg et al., 2007; Wu and Porté-Agel, 2012). According to their investigations, the wake structure is strongly influenced by the presence of turbulence in the inflow and the wake recovers more rapidly for higher turbulence intensity levels of the incoming flow.

Different methods have been applied to generate a turbulent flow field upstream of the wind turbine. In wind-tunnel experiments, additional roughness elements in front of the wind turbine evoke a turbulent flow, which can be generated by turbulence grids (Medici and Alfredsson, 2006) or obstacles on the floor (Chamorro and Porté-Agel, 2009). Implementing this method in a numerical simulation requires a rather large upstream section, which is computationally expensive, leading to other approaches.

A simple synthetic method avoiding the simulation of atmospheric turbulence was proposed by Mann (1994), e.g. used in Troldborg et al. (2007). The resulting three-dimensional turbulence field is compact and provides turbulence spectra as expected in an ABL. This method, however, is not based on a physical model and only offers a synthetic turbulence field (Naughton et al., 2011). An alternative approach is to couple meteorological data (e.g. wind speed, wind direction, temperature) from a mesoscale simulation on the microscale LES of the wind turbine. However, the two-way coupling as well as the one-way coupling between mesoscale and microscale models, induces different problems (Mirocha et al., 2013; Muñoz-Esparza et al., 2014).

The necessity of synthetic or mesoscale atmospheric parameters can be avoided by the use of a precursor simulation. Wu and Porté-Agel (2012) created a neutral ABL flow forced by a streamwise pressure gradient. The main simulation is initialized with data from the precursor simulation. By applying streamwise periodic boundary conditions, a buffer zone prevented the turbulence in the wake from re-entering the domain and interacting with the wind turbine. 
Open streamwise boundary conditions do not require a buffer zone. Instead, the wind-turbine simulation has to be fed continuously with turbulence data from a precursor simulation to generate a fully developed turbulent flow field. Naughton et al. (2011) ensured a turbulent inflow by prescribing instantaneous velocity components from the precursor simulation at the inflow plane at regular time intervals. Witha et al. (2014) realized a turbulent inflow for an array of wind turbines in a wind park based on a recycling method after Kataoka and Mizuno (2002). The main simulation used the data from the precursor simulation for initialization and persistently extracted turbulence from a region upstream of the wind turbine adding it to the mean inflow profiles.

The first goal of our study is to develop and investigate a new methodology to generate and maintain a realistic background turbulence field in the wind-turbine LES with open horizontal boundary conditions, and by avoiding a continuous turbulent inflow from a precursor simulation. At each timestep of the wind-turbine LES, the flow field shall be perturbed by velocity fluctuations extracted from a selected state of the precursor simulation of a neutral ABL. The aim is to maintain the spectral properties of realistic background turbulence and to control the energy of the applied perturbation fields. Here, we describe the new methodology and compare our numerical results with published results from previous simulations and measurements.

In addition to a realistic background turbulence field, an LES of windturbine wakes require a detailed knowledge and parametrization of the forces exerted by a wind turbine on the atmosphere. In a numerical model the windturbine forces can be parametrized as a disc that can either rotate or not. Alternatively, individual rotating lines represent the blades of the wind turbine. The respective approaches are termed the actuator disc model (ADM) and the actuator line model (ALM). The impact of wind-turbine parametrizations on the wake has been studied focusing on various aspects.

Mikkelsen (2003) investigated the parametrization of a wind turbine with the ADM and the ALM, extended for a multiplicity of rotor configurations, e.g. a coned or a yawed rotor. Numerous investigations validating the different wind-turbine parametrizations were performed by e.g. Ivanell et al. (2008), Porté-Agel et al. (2010), Wu and Porté-Agel (2011) and Tossas and Leonardi (2013). All of these studies resulted in a near-wake wind field, sensitive to the wind-turbine parametrization, whereas the far-wake structure depends mainly on the background turbulence. Mirocha et al. (2014) implemented the generalized actuator disc wind-turbine parametrization into the Weather Research and Forecasting (WRF-LES) model. This approach enabled the investigation of the interaction of a wind turbine with different ABL stratifications, resulting in good agreement of the wake characteristics with observations under weakly convective conditions. Numerous studies explored the impact of the distribution of the forces. Ivanell et al. (2008) and Tossas and Leonardi (2013) studied the impact of different smearing parameters of the forces acting on the atmosphere, resulting in numerical instabilities for a tight volume-force distribution at the rotor position. Ivanell et al. (2008), Wu and Porté-Agel (2011) and Gomes et al. (2014) investigated the influence of the number of 
grid points representing the disc on the wake structure with the result that the wake characteristics are independent of the resolution, if a minimum of ten grid points cover the rotor diameter in the spanwise and the vertical directions. Gomes et al. (2014) also analyzed the effect of the radial dependencies of the applied forces. A strong sensitivity of the near-wake wind field was found in contrast to the far-wake behaviour.

Here, we apply a modified version of the classical Rankine-Froude ADM and the blade element momentum (BEM) method for two different airfoils as wind-turbine parametrizations in our numerical simulations. In the second part, systematic investigations of the wake characteristics depending on the two parametrizations, the local blade characteristics, and the rotation of the disc are made.

We implement our turbulence preserving method and both wind-turbine parametrizations in the multiscale geophysical flow solver EULAG (Prusa et al., 2008). This LES model resolves all energy containing modes of the turbulent transport and scales larger than the spatial resolution of the computational grid. Only the turbulence of the smallest unresolved scales is parametrized using a subgrid-scale (SGS) closure model. The sensitivity of the numerical results towards different SGS closure models (turbulent kinetic energy (TKE) closure, Smagorinsky closure) as well as an implicit LES (Grinstein et al., 2007) constitute the third task investigated.

The outline of the paper is as follows: the LES model is presented in Sect. 2, while the turbulence preserving method is formulated in Sect. 3, and the windturbine models are described in Sect. 4 . The results of the numerical simulations studying the influence of the intensity of background turbulence, the wind-turbine parametrizations, the rotation of the wind turbine and the SGS closure models on the wake characteristics follow in Sect. 5. Conclusions are given in Sect. 6.

\section{Numerical model framework}

An inviscid and incompressible flow through a wind turbine is simulated with the multiscale geophysical flow solver EULAG (Prusa et al., 2008). The geophysical flow solver EULAG is at least second-order accurate in time and space (Smolarkiewicz and Margolin, 1998) and is well suited for massively-parallel computations (Prusa et al., 2008). It can be run parallel up to a domain decomposition in three dimensions. A comprehensive description and discussion of the geophysical flow solver EULAG can be found in Smolarkiewicz and Margolin (1998) and Prusa et al. (2008).

For the numerical simulations conducted herein, the Boussinesq equations for a flow with constant density $\rho_{0}=1.1 \mathrm{~kg} \mathrm{~m}^{-3}$ are solved for the Cartesian velocity components $\mathbf{v}=(u, v, w)$ and for the potential temperature pertur- 
bations $\Theta^{\prime}=\Theta-\Theta_{0}$ (Smolarkiewicz et al., 2007),

$$
\begin{aligned}
\frac{d \mathbf{v}}{d t} & =-G \boldsymbol{\nabla}\left(\frac{p^{\prime}}{\rho_{0}}\right)+\mathbf{g} \frac{\Theta^{\prime}}{\Theta_{0}}+\mathcal{V}+\mathbf{M}+\frac{\mathbf{F}}{\rho_{0}} \equiv \mathcal{R}^{\boldsymbol{v}}, \\
\frac{d \Theta^{\prime}}{d t} & =\mathcal{H} \equiv \mathcal{R}^{\Theta}, \\
\nabla \cdot\left(\rho_{0} \mathbf{v}\right) & =0,
\end{aligned}
$$

where $\Theta_{0}=301 \mathrm{~K}$. In Eqs. 1,2 , and $3, d / d t, \boldsymbol{\nabla}$, and $\boldsymbol{\nabla} \cdot$ represent the total derivative, the gradient and the divergence, respectively. The quantity $p$ 'represents the pressure perturbation with respect to the environmental state and $\mathrm{g}$ is the vector of acceleration due to gravity. The factor $G$ represents geometric terms that result from the general, time-dependent coordinate transformation (Wedi and Smolarkiewicz, 2004; Smolarkiewicz and Prusa, 2005; Prusa et al., 2008; Kühnlein et al., 2012). The SGS terms $\mathcal{V}$ and $\mathcal{H}$ symbolise viscous dissipation of momentum and diffusion of heat, $\mathbf{M}$ denotes the inertial forces of coordinate-dependent metric accelerations and $\mathbf{F}$ additional external forces related to the parametrization of the wind turbine in the geophysical flow solver EULAG. The terms $\boldsymbol{\mathcal { R }}^{\boldsymbol{v}}$ and $\mathcal{R}^{\Theta}$ summarize symbolically all forces in the corresponding equations.

The acronym EULAG refers to the ability of solving the equations of motions either in an EUlerian (flux form) (Smolarkiewicz and Margolin, 1993) or in a semi-LAGrangian (advective form) (Smolarkiewicz and Pudykiewicz, 1992) mode, via

$$
\psi^{\xi+1}=\mathrm{LE}\left(\psi^{\xi}+\left.\frac{1}{2} \Delta t \mathcal{R}^{\psi}\right|^{\xi}\right)+\left.\frac{1}{2} \Delta t \mathcal{R}^{\psi}\right|^{\xi+1}
$$

where $\psi=(u, v, w, \Theta), \xi$ denotes the timestep and LE is the corresponding finite-difference operator (semi-Lagrangian/Eulerian). In general, the geophysical flow solver EULAG owes its versatility to a unique design that combines a rigorous theoretical formulation in generalized curvilinear coordinates (Smolarkiewicz and Prusa, 2005) with non-oscillatory forward-in-time (NFT) differencing for fluids built on the multi-dimensional positive definite advection transport algorithm (MPDATA), which is based on the convexity of upwind advection (Smolarkiewicz and Margolin, 1998; Prusa et al., 2008) and a robust, exact-projection type, elliptic Krylov solver (Prusa et al., 2008). The flow solver has been applied to a wide range of scales simulating various problems like turbulence (Smolarkiewicz and Prusa, 2002), flow past complex or moving boundaries (Wedi and Smolarkiewicz, 2006; Kühnlein et al., 2012), gravity waves (Smolarkiewicz and Dörnbrack, 2008; Doyle et al., 2011) or even solar convection (Smolarkiewicz and Charbonneau, 2013). The turbulence closure in the geophysical flow solver EULAG can be described by a TKE model, a Smagorinsky model or an implicit LES, with no turbulence closure model due 
to not considering the diffusion process. The implicit LES properties of numerical solvers based on MPDATA are documented in e.g. Margolin and Rider (2002), Margolin et al. (2002) and Margolin et al. (2006) for structured grids. A detailed description of an implicit LES is given in Grinstein et al. (2007).

\section{Turbulence preserving method}

The basic idea of our new methodology that preserves the background turbulence in an LES of a flow through a wind turbine is to extract velocity perturbations from a precursor simulation of the neutral ABL. The velocity fields are used to disturb the wind-turbine simulation in a special manner as described below. For this purpose, a precursor simulation of the turbulent neutral ABL has to be conducted.

\subsection{Precursor simulation}

To drive the neutral ABL flow, an additional forcing $-u_{*}^{2} / H$ is applied for the $u$-component of Eq. 1, where $H$ is the height of the computational domain. Sensitivity tests revealed that a value of the friction velocity $u_{*}=0.4 \mathrm{~m} \mathrm{~s}^{-1}$ results in a realistic pressure gradient of the ABL. This forcing is comparable to the streamwise mean pressure gradient force applied in Wu and Porté-Agel (2012). The precursor simulation is performed with the same number of grid points as the wind-turbine simulations, but with periodic boundary conditions in the horizontal directions. The initial wind speed is set to zero, and the drag coefficient in the surface parametrization is set to 0.1 .

Applying only the above forcing, it is a long lasting process until the precursor simulation is in an equilibrium state. Additional velocity gradients in the neutral flow can serve as a trigger, breaking the symmetry and acting as a seed for turbulence to develop. Therefore, the precursor simulation is disturbed by inserting an obstacle in the domain for a few timesteps. The flow around this obstacle enhances the velocity gradients in the neutral ABL flow, and the equilibrium state of the precursor simulation is attained more rapidly.

\subsection{Methodology}

The perturbation velocities $\left.\mathbf{u}_{p}^{*}\right|_{i, j, k} ^{\xi}$ are extracted from the precursor simulation according to,

$$
\left.\mathbf{u}_{p}^{*}\right|_{i, j, k} ^{\xi}=\alpha \cdot \beta \cdot \underbrace{(\left.\mathbf{u}_{p}\right|_{i^{*}, j, k}-\underbrace{\left.\frac{1}{n \cdot m} \sum_{i=1}^{n} \sum_{j=1}^{m} \mathbf{u}_{p}\right|_{i, j, k}}_{I})}_{I I},
$$


where $\left.\mathbf{u}_{p}\right|_{i^{*}, j, k}$ is the velocity vector of the precursor simulation in an equilibrium state and the term $I$ in Eq. 5 denotes the height-averaged mean value of the corresponding wind component at each grid point $i, j$, and $k$. The indices of the grid points are denoted by $i=1 \ldots n, j=1 \ldots m$, and $k=1 \ldots l$ in the $x, y$, and $z$ directions, respectively.

The perturbation velocity from Eq. 5 contributes to the velocity field of the wind-turbine simulation $\left.\mathbf{u}\right|_{i, j, k} ^{\xi}$ at the initial timestep $\xi=0$ and at each following timestep $\xi$. The values of the precursor simulation $\left.\mathbf{u}_{p}\right|_{i^{*}, j, k}$ are shifted in the streamwise direction by one grid point every timestep $\xi$, symbolized by $i^{*}=i+\xi^{*}$, with $i^{*} \in[1, n]$ and $\xi^{*}$ representing the number of timesteps since the start of the simulation. Furthermore, the difference as denoted by $I I$ in Eq. 5 is multiplied with a random number $\beta$ ranging from -0.5 to 0.5 . Both the grid point shift and the random number multiplication are necessary to only apply the spectral energy distribution of the precursor simulation instead of impressing individual flow patterns onto the wind-turbine simulation. To account for different magnitudes of the background turbulence, the term $I I$ in Eq. 5 is additionally multiplied by a factor $\alpha$, representing the amplitude of the turbulence perturbations (hereafter referred to as perturbation amplitude).

Applying this method maintains the spectral properties of the turbulent fluctuations in the wind-turbine simulation. It offers several possibilities for the numerical scheme:

1. Periodic boundary conditions and a buffer zone can be avoided, enabling open inflow and outflow Neumann boundary conditions and minimising the domain size of the simulation.

2. The perturbation data from the precursor simulation are imported only once and are stored in three $3 \mathrm{D}$ fields $(u, v, w)$ during the wind-turbine simulation.

3. The method is computationally very efficient, as it allows to reapply the background turbulence of one precursor simulation to a variety of windturbine simulations.

4. The response of a wind turbine to different intensities of the background turbulence can be easily investigated by changing the parameter $\alpha$ in Eq. 5 .

\subsection{Validation of the turbulence preserving method}

We performed a simulation applying term $I$ from Eq. 5 as wind field. In addition, the spectral energy distribution of the precursor simulation is applied with the prescribed methodology. After integrating for the same amount of time as in the following wind-turbine simulations, this simulation resulted in the same values of $\langle u\rangle_{t},\langle v\rangle_{t}$ and $\langle w\rangle_{t}$, as well as $\sigma_{u}, \sigma_{v}$ and $\sigma_{w}$ with $\sigma_{i}=\sqrt{i^{\prime 2}}$ as the precursor simulation, validating the mechanism of the turbulence preserving method. 


\section{Wind-turbine Parametrization}

\subsection{Parametrization of the forces}

The classical Rankine-Froude theory is the simplest ADM representation of turbine-induced forces in a numerical model where the disc covers the span of the blades. It was introduced by Froude (1889) who continued the work of Rankine (1865) on the momentum theory of propellers. The forces induced by a wind turbine are basically parametrized as a $1 \mathrm{D}$ thrust force, which is constant over the disc. Despite its simplicity, this non-rotating ADM has been widely used in LES as it provides reliable results on coarse grids (Calaf et al., 2010; Porté-Agel et al., 2010; Wu and Porté-Agel, 2011; Tossas and Leonardi, 2013; Meyers and Meneveau, 2013). A wind turbine rotates and the incoming profiles of the horizontal wind speed are often vertically sheared $(\partial \mathbf{u} / \partial z \neq 0)$. Both processes limit the applicability of the simple ADM parametrization. To circumvent these limitations and to enable an investigation of the impact of the local blade characteristics by comparing to the results of the BEM parametrization (Manwell et al., 2002; Hansen, 2008), we apply a modified version of the Rankine-Froude ADM considering the axial force $F_{x}(y, z)$ in the streamwise $(x)$ direction and the tangential force $F_{\Theta}(y, z)$ perpendicular to $F_{x}$ in the $y$ - $z$ plane,

$$
\begin{aligned}
\left.\left|F_{x}\right|\right|_{x_{0}, y, z} & =\frac{1}{2} \rho_{0} c_{T}^{\prime} A_{x_{0}, y, z}<u_{x_{0}, y, z}^{2}>_{t} \\
\left.\left|F_{\Theta}\right|\right|_{x_{0}, y, z} & =\frac{1}{2} \rho_{0} c_{P}^{\prime} A_{x_{0}, y, z}<u_{x_{0}, y, z}^{2}>_{t} \frac{u_{x_{0}, y, z}}{\Omega r_{x_{0}, y, z}}
\end{aligned}
$$

Both forces $F_{x}$ and $F_{\Theta}$ result in the total force $\left.\mathbf{F}\right|_{x_{0}, y, z}$ (Hansen, 2008), with

$$
\left.\mathbf{F}\right|_{x_{0}, y, z}=\left.\mathbf{F}_{x}\right|_{x_{0}, y, z}+\left.\mathbf{F}_{\Theta}\right|_{x_{0}, y, z},
$$

where the centre of the rotor is defined by the grid-point coordinates $x_{0}, y_{0}$ and $z_{h}$ (hub height). In Eqs. 6 and $7, c_{T}$ represents the thrust coefficient $\left(c_{T}^{\prime}=c_{T} /(1-a)^{2}\right)$ and $c_{P}$ the power coefficient $\left(c_{P}^{\prime}=c_{P} /(1-a)^{3}\right)$. The factor $a$ corresponds to the axial induction factor and can be derived from the onedimensional momentum theory to a value of $1 / 3$ for an ideal rotor (Betz, 1926). $A_{x_{0}, y, z}$ is the area of the rotor at position $x_{0}$ covered by grid points in the $y-z$ plane, $\Omega$ is the angular velocity of the turbine and $r_{x_{0}, y, z}$ the radial position inside the rotor $\left(0 \leq r_{x_{0}, y, z} \leq R\right)$, with $R=D / 2$ and $D$ representing the diameter of the wind-turbine rotor. The time-averaged value of the squared streamwise velocity component at the rotor position $x_{0}, y, z$ is denoted by $<u_{x_{0}, y, z}^{2}>_{t}$.

A great improvement of the simple momentum theory was the classical BEM method by Glauert (1963). This method accounts for local blade characteristics, as it enables calculation of the steady loads as well as the thrust and the power for different wind speeds, rotational speeds, and pitch angles of 
Table 1 The crucial characteristics of the three different parametrizations A, B, and C of the wind turbine used in this study.

\begin{tabular}{c|c|c} 
Parametrization & $\mathrm{A}$ & $\mathrm{B}+\mathrm{C}$ \\
\hline Name & MMT & BEM \\
\hline Characteristics & Eq. 6 & Eq. 9 \\
& Eq. 7 & Eq. 10 \\
\hline
\end{tabular}

the blades. The axial and tangential forces of the BEM method are represented in Eqs. 9 and 10,

$$
\begin{aligned}
\left.\left|F_{x}\right|\right|_{x_{0}, y, z}= & \frac{1}{2} \rho_{0} \frac{B c}{2 \pi r_{x_{0}, y, z}}\left(c_{L} \cos \Phi+c_{D} \sin \Phi\right) \\
& \times A_{x_{0}, y, z} \frac{u_{x_{\infty}, y, z}^{2}(1-a)^{2}}{\sin ^{2} \Phi} \\
\left|F_{\Theta}\right|_{x_{0}, y, z}= & \frac{1}{2} \rho_{0} \frac{B c}{2 \pi r_{x_{0}, y, z}}\left(c_{L} \sin \Phi-c_{D} \cos \Phi\right) \\
& \times A_{x_{0}, y, z} \frac{u_{x_{\infty}, y, z}(1-a) \Omega r_{x_{0}, y, z}\left(1+a^{\prime}\right)}{\sin \Phi \cos \Phi} .
\end{aligned}
$$

Here, $B$ represents the number of blades, $c$ is the chord length of the blade, $c_{L}$ is the lift coefficient, $c_{D}$ is the drag coefficient, $\Phi$ is the angle between the plane of rotation and the relative streamwise velocity, and $a^{\prime}$ is the tangential induction factor. Following Hansen (2008), we calculate $a$ and $a^{\prime}$ by an iterative procedure from the airfoil data. The upstream velocity $u_{x_{\infty}, y, z}$ is taken at the first upstream grid point in the $x$-direction and the corresponding $y$ and $z$ coordinates. With the exception of $\rho_{0}$ and $B$, all other parameters appearing in Eqs. 9 and 10 depend on the radius $r_{x_{0}, y, z}$ and vary spatially.

In this work, the modified version of the Rankine-Froude ADM as well as the BEM parametrization are implemented via Eq. 8 in the geophysical flow solver EULAG. The forces are treated implicitly in the numerical scheme according to Eq. 4. In the geophysical flow solver EULAG, the rotor of a wind turbine is not implemented as a real circular obstacle (e.g. grid-point blocking as in Heimann et al. (2011)) or a permeable rotor (Witha et al., 2014; Tossas and Leonardi, 2013; Gomes et al., 2014). Instead, at every grid point covered by the rotor, the velocity field experiences the turbine-induced force $\mathbf{F}$ according to Eq. 1. This implementation is inspired by the immersed boundary method, successfully applied in the geophysical flow solver EULAG by Smolarkiewicz and Winter (2010). The implicit treatment of the forces in Eq. 4 has a positive effect on the timestep, because there are no large velocity gradients between the rotor area and its surroundings.

Altogether, three different parametrizations of wind-turbine induced forces are implemented in the geophysical flow solver EULAG. The respective parametrizations $\mathrm{A}, \mathrm{B}$, and $\mathrm{C}$ are listed together with their main characteristics in Table 1. It should be noted that the parametrizations B and $\mathrm{C}$ are essentially the same, however, the airfoil data applied in $\mathrm{B}$ and $\mathrm{C}$ differ. The radial distri- 


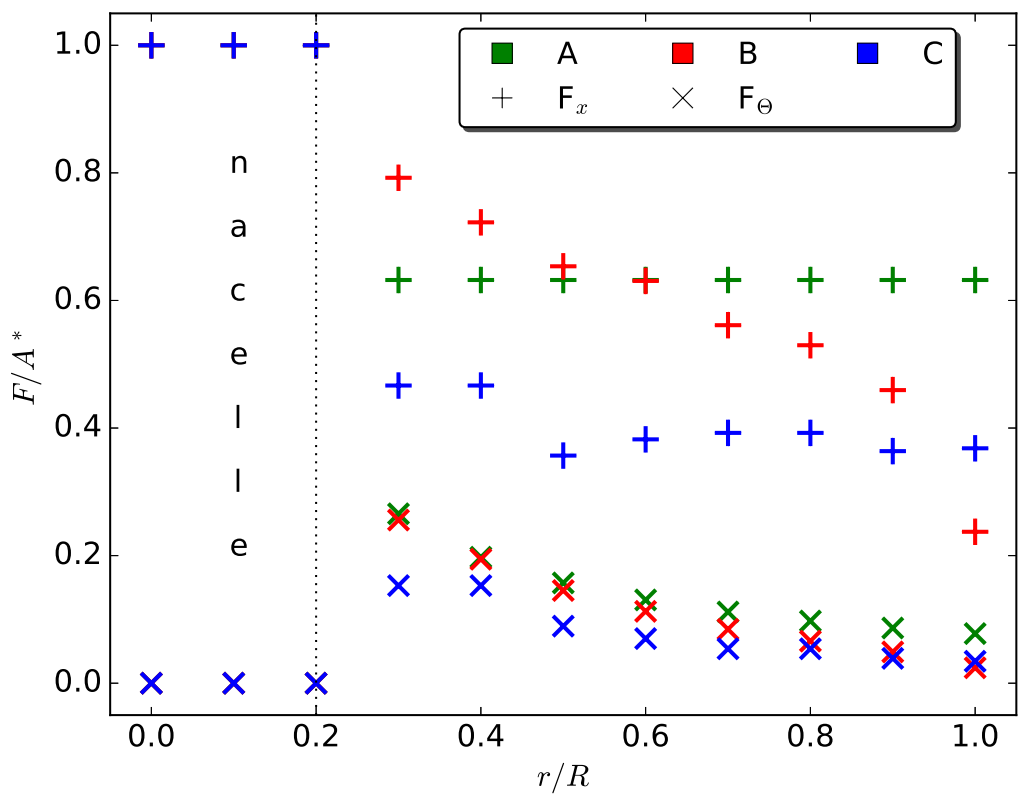

Fig. 1 Radial distributions of the axial and tangential forces $F_{x}$ and $F_{\Theta}$ normalized by the area $\mathrm{A}^{*}$ for the different wind-turbine parametrizations $\mathrm{A}, \mathrm{B}$, and $\mathrm{C}$ of Table 1 . The values of $F_{x}$ and $F_{\Theta}$ are normalized by the maximum of the axial force at the nacelle, which is the same in all three parametrizations. The axial forces are represented by $(+)$ and the tangential forces by $(x)$. They are plotted for each discrete position of the rotor, assuming 21 grid points are covering the rotor with radius $R$. The nacelle covers $20 \%$ of the blades, denoted by the dotted vertical line. For the calculation of the forces in these schematic illustration, a rotor diameter of $100 \mathrm{~m}$ is assumed, together with a rotation frequency $\Omega=7$ r.p.m. and a constant upstream velocity $u_{x_{\infty}, y, z}=8 \mathrm{~m} \mathrm{~s}^{-1}$.

butions of the respective axial and tangential forces are depicted in Fig. 1. In each parametrization, a nacelle is represented within $r / R \leq 0.2$ by a stronger drag force in comparison to the blade values and no lift force. The size of the parametrized nacelle is large compared to a real wind turbine, because the numerical resolution demands enough grid points representing the nacelle to avoid instabilities. The tower is not considered in our parametrizations as it is not the major source of turbulence.

Parametrization A represents the modified version of the Rankine-Froude $\mathrm{ADM}$, hereafter referred to as modified momentum theory (MMT). It can be applied for a rotating actuator with $F_{\Theta} \neq 0$ or for a non-rotating actuator with $F_{\Theta}=0$. Parametrization A can be regarded as a simplified version of parametrization $\mathrm{B}$, as the values of $\mathrm{c}_{T_{\text {blade }}}^{\prime}=1.27$ and $\mathrm{c}_{P_{\text {blade }}}^{\prime}=0.87$ in Eqs. 6 and 7 are deduced from parametrization B. These prescribed values are comparable to other studies (Meyers and Meneveau, 2013). 
The BEM method is used to investigate the influence of the blade structure. 342 The airfoil data are taken from two different wind turbines. The $10 \mathrm{MW}$ refer-

ence wind turbine from DTU (Technical University of Denmark) referred to as parametrization B (Mark Zagar (Vestas), personal communication, 2015) and the three-blade GWS/EP-6030x3 rotor (Wu and Porté-Agel, 2011) referred to as parametrization C. For both wind turbines, the rotor radius as well as the chord length of the blades are scaled to a rotor diameter of $100 \mathrm{~m}$, to make the results comparable to each other. The most relevant wind-turbine parameters used for parametrizations $\mathrm{B}$ and $\mathrm{C}$ are listed in the Appendix.

For the nacelle, $\mathrm{c}_{T_{\text {nacelle }}}^{\prime}=1.48$ and $\mathrm{c}_{P_{\text {nacelle }}}^{\prime}=0$ are chosen in all three parametrizations. The value of the drag coefficient of the nacelle of 1.0 agrees with the drag coefficient interval of cylindrically shaped bluff bodies between 0.8 and 1.2 (Schetz and Fuhs, 1996), and has also been used e.g. in El Kasmi and Masson (2008).

\subsection{Application of the forces}

The numerical simulations conducted in this study are performed on an equidistant Cartesian mesh with grid spacings $\Delta x, \Delta y$ and $\Delta z$, in the streamwise, lateral and vertical directions, respectively. It must be noted, that all parametrizations A, B, and C are coded to perform properly in terrain-following coordinates with variable vertical grid spacings over hilly terrain.

To calculate the forces of the actuator, we use polar coordinates that serve as a local mesh. The centre coordinate of the polar mesh is the centre of the rotor. From this position, the polar mesh is described by a very fine grid with $\Delta r=R / 1000$ as radial step size and $\Delta \varphi=1^{\circ}$ as azimuthal step size. The step sizes in the radial and azimuthal directions are fine enough to minimize the errors that would result from calculating the forces on a Cartesian mesh (Ivanell et al., 2008). The computational costs arising from such a fine polar mesh are insignificant, as the disc is always at the same position, making this calculation of the actuator force in polar coordinates $F_{r, \Theta, z}$ only necessary once.

The force acting on each polar grid point $F_{r, \Theta, z}$ is transformed to the corresponding force in Cartesian coordinates $F_{x, y, z}^{*}=\mathcal{M}_{x, y, z} \cdot F_{r, \Theta, z}$ through the transformation matrix $\mathcal{M}_{x, y, z}$. The force $F_{x, y, z}^{*}$ contributes to a certain fraction $\mu \in[0,1]$ to the actuator force $F_{x, y, z}=\mu \cdot F_{x, y, z}^{*}$. The fraction $\mu$ is determined by the ratio of the grid-cell volume of the polar coordinate and the corresponding Cartesian coordinate, i.e. $\mu=1$ if the Cartesian grid point is completely covered by the rotor and $\mu=0$ in case of a rotor-free grid point. At the edge of the rotor, the fraction $\mu<1$, because the Cartesian grid cell is not completely covered by the local polar mesh representing the rotor.

A smearing of the turbine-induced forces in the axial as well as in the radial direction is necessary to avoid numerical instabilities. As a first step, the forces from Eq. 8 are additionally distributed in the streamwise direction. This approach is performed for all parametrizations. The forces in Eq. 8 are 
smeared with a $1 \mathrm{D}$ Gaussian function in the $x$-direction,

$$
F_{s_{x}}=\frac{1}{\sqrt{\pi} \sigma} \exp \left(-\frac{\left(x-x_{0}\right)^{2}}{\sigma^{2}}\right) .
$$

Similar to other studies (Meyers and Meneveau, 2013), the value of $\sigma$ is set to 1.5 and is given in absolute values of the radius.

In parametrization $\mathrm{A}$, the axial force $F_{x}$ in the $y$ - $z$ plane only varies with the incoming velocity across the rotor. A moderate velocity gradient results in very similar $F_{x}$ values and generates large gradients at the edges of the rotor. An additional two-dimensional smearing $F_{s_{y-z}}$ in the $y$ - $z$ plane is introduced to avoid too sharp radial gradients in the turbine-induced forces between the rotor area and the immediate surroundings. The forces of the schematic illustration in Fig. 1 decrease with a step function over the last three grid points $\in[0.8 \mathrm{r} / R, 1.0 \mathrm{r} / R]$. The force at each of these outer region grid points is half of the force of the corresponding nearest inner neighbour grid point. $F_{s_{y-z}}$ is not applied for the forces in the BEM method, as the parameters in Eqs. 9 and 10 already decrease with increasing $r$.

The values of the smearing parameters and of the step function applied on the forces in the $y-z$ plane in parametrization A are chosen in such a way that the integrated force distributed in three dimensions is the same as in the two-dimensional case without smearing. By combining the smearing in the $x$ direction $F_{s_{x}}$ and the smearing in the $y$ - $z$ plane $F_{s_{y-z}}$, the difference of the forcings between a $2 \mathrm{D}$ and a $3 \mathrm{D}$ disc is less than $1 \%$ for 21 grid points per disc and decreases for a finer resolution.

The parametrization $\left.\mathbf{F}\right|_{x_{0}, y, z}$ (Eq. 8) together with the coordinate transformation $F_{x, y, z}$ and the applied smearing in the axial $F_{s_{x}}$ and radial $F_{s_{y-z}}$ directions result in a total parametrized force,

$$
\left.\mathbf{F}\right|_{x, y, z}=\left.\mathbf{F}\right|_{x_{0}, y, z} \cdot F_{x, y, z} \cdot F_{s_{x}} \cdot F_{s_{y-z}},
$$

where the wind-turbine induced force $\left.\mathbf{F}\right|_{x, y, z}$ corresponds to the force $\mathbf{F}$ in Eq. 1.

\subsection{Validation of the wind-turbine parametrization}

We validate our numerical results for the wind-turbine parametrizations $\mathrm{A}$ $\mathrm{B}$, and $\mathrm{C}$ at the rotor position $\left(x_{0}, y, z\right)$ and in the wake $\left(x_{w}, y, z\right)$, whereby $x_{w} \geq x_{0}$, with theoretical wind predictions from the one-dimensional momentum theory,

$$
\begin{aligned}
u_{x_{0}, y, z} & =u_{x_{\infty}, y, z}(1-a), \\
u_{x_{w}, y, z} & =u_{x_{\infty}, y, z}(1-2 a),
\end{aligned}
$$

where $a$ is the axial induction factor defined as

$$
a:=\frac{u_{x_{\infty}, y, z}-u_{x_{0}, y, z}}{u_{x_{\infty}, y, z}} .
$$


Table 2 Parameters for the two different wind turbines (wind turbine 1 in laminar flow and wind turbine 2 in the turbulent ABL) with the rotor diameter $D$, the hub height $z_{h}$, the spatial resolution $\Delta$, the rotation frequency of the blades $\Omega$ in revolutions per minute (r.p.m.), the location of the centre of the rotor in the simulated domain, as well as the velocity at the hub height $u_{x, y, z_{h}}$ of the wind turbine and the vertical profile of the incoming velocity $u_{x_{\infty}, y, z}$. In the prescribed logarithmic wind profile, $u_{*}$ represents the friction velocity, $\kappa$ is the von Karman constant $(\kappa=0.4)$, and $z_{0}$ is the roughness length. All simulations are performed on an equidistant grid with the spacing $\Delta=\Delta x=\Delta y=\Delta z$.

\begin{tabular}{c|c|c} 
parameters & wind turbine 1 & wind turbine 2 \\
\hline grid points & $512 \times 128 \times 128$ & $512 \times 64 \times 64$ \\
\hline$D(\mathrm{~m})$ & 4 & 100 \\
\hline$z_{h}(\mathrm{~m})$ & 4 & 100 \\
\hline$\Delta(\mathrm{m})$ & 0.1 & 5 \\
\hline$\Omega(\mathrm{r} . \mathrm{p} . \mathrm{m})$. & 0 & $x_{0}=60 \Delta$ \\
\hline rotor centre & $x_{0}=120 \Delta$ & $y_{0}=32 \Delta$ \\
& $y_{0}=64 \Delta$ & $z_{h}=20 \Delta$ \\
\hline$u_{x, y, z_{h}}\left(\mathrm{~m} \mathrm{~s}^{-1}\right)$ & 0.08 and 0.10 & 8.0 \\
\hline$u_{x_{\infty}, y, z}$ & constant wind profile & logarithmic wind profile \\
& $\frac{d u}{d z}=0$ & $u_{x_{\infty}, y, z}=\frac{u_{*}}{\kappa} \ln \left(\frac{z}{z_{0}}\right)$ \\
& & $u_{*}=0.45 \mathrm{~m} \mathrm{~s} \mathrm{~s}^{-1} ; z_{0}=0.1 \mathrm{~m}$ \\
\hline
\end{tabular}

Table 3 Theoretically predicted velocities for different axial induction factors $a$ at the rotor position $u_{x_{0}, y, z}$ and in the wake $u_{x_{w}, y, z}$ scaled with the upstream velocity $u_{x_{\infty}, y, z}$ according to Eqs. 13 and 14 and the deviations obtained from the numerical simulations. The deviations are calculated as an average over the disc area.

\begin{tabular}{c|c|c|c|c}
$a$ & $\frac{u_{x_{0}, y, z}}{u_{x_{\infty}, y, z}}$ expected & $\frac{u_{x_{0}, y, z}}{u_{x_{\infty}, y, z}}$ deviation & $\frac{u_{x_{w}, y, z}}{u_{x_{\infty}, y, z}}$ expected & $\frac{u_{x_{w}, y, z}}{u_{x_{\infty}, y, z}}$ deviation \\
\hline $1 / 3$ & 0.67 & $2 \%$ & 0.33 & $5 \%$ \\
\hline $1 / 4$ & 0.75 & $0 \%$ & 0.50 & $0 \%$ \\
\hline $1 / 5$ & 0.80 & $2 \%$ & 0.60 & $4 \%$ \\
\hline
\end{tabular}

Equation 13 follows directly from Eq. 15, and Eq. 14 can be derived from the Bernoulli equation and Newton's second law of motion (Hansen, 2008). This comparison is strictly applicable only for laminar and uniform inflow conditions $u_{x_{\infty}, y, z}$.

Numerical simulations with the set-up as listed in Table 2 for wind turbine 1 are performed with different axial induction factors $a=1 / 3,1 / 4,1 / 5$ for all parametrizations. Exemplary, the results for parametrization A, a nonrotating disc and $u_{x_{\infty}, y, z}=0.08 \mathrm{~m} \mathrm{~s}^{-1}$ are listed in Table 3 . The results for parametrizations $\mathrm{B}$ and $\mathrm{C}$ and for $u_{x_{\infty}, y, z}=0.10 \mathrm{~m} \mathrm{~s}^{-1}$ are quantitatively similar and therefore not shown here.

The simulated ratios of $u_{x_{0}, y, z} / u_{x_{\infty}, y, z}$ and $u_{x_{w}, y, z} / u_{x_{\infty}, y, z}$ for a realistic value of the axial induction factor of $1 / 4$ are in complete agreement with the one-dimensional momentum theory. For larger $(a=1 / 3)$ and smaller $(a=1 / 5)$ $a$ values, the simulation results deviate by less than $5 \%$ from the theoretical predictions. 
Table 4 List of all performed simulations with information of the perturbation amplitude, the type of the wind-turbine parametrization, the tangential force and the SGS closure model used in the LES model.

\begin{tabular}{c|c|c|c|c} 
simulation & $\begin{array}{c}\text { perturbation } \\
\text { amplitude } \alpha\end{array}$ & $\begin{array}{c}\text { wind turbine } \\
\text { parametrization }\end{array}$ & $\begin{array}{c}\text { tangential } \\
\text { force } F_{\Theta}\end{array}$ & $\begin{array}{c}\text { SGS } \\
\text { closure model }\end{array}$ \\
\hline B_1 & 1 & B & $\neq 0$ & TKE \\
\hline B_5 & 5 & B & $\neq 0$ & TKE \\
B_10 & 10 & B & $\neq 0$ & TKE \\
\hline A_1 & 1 & A & $\neq 0$ & TKE \\
C_1 & 1 & C & $\neq 0$ & TKE \\
\hline A_NR & 1 & A & $\neq 0$ & TKE \\
\hline B_S & 1 & B & $\neq 0$ & Smagorinsky \\
B_I & 1 & B & $\neq 0$ & no (implicit LES) \\
\hline
\end{tabular}

Summarizing, we successfully validated our LES model EULAG for the non-rotating disc of parametrization $\mathrm{A}$ and realistic values of the axial induction factor against the one-dimensional momentum theory.

\section{Numerical Experiments and Results}

In this section, a detailed investigation of the reference simulation B_1 (base case) with $\alpha=1$ and wind turbine 2 (Table 2) is given to confirm the application of the turbulence preserving model in a wind-turbine simulation. Details of the simulation set-up are listed in Table 2. Further, the dependence of the wake characteristics of the reference simulation $B_{-} 1$ are investigated regarding the impact of,

$a$, the perturbation amplitude

$b$, the wind-turbine parametrization

$c$, the rotation of the disc

$d$, the SGS closure model.

The corresponding parameters of B_1 and of all other simulations are listed in Table 4.

All simulations are performed for $60 \mathrm{~min}$, a period long enough for the wake to reach an equilibrium state with statistical convergence of the results. All mean values are averaged over the last $50 \mathrm{~min}$. The temporal average $\left\langle\Psi_{x, y, z}\right\rangle_{t}$ of a quantity $\Psi$ for a time period $t$ is calculated online in the numerical model and updated at every timestep according to the method of Fröhlich (2006, Eq. 9.1). In the following numerical simulations, the rotor covers 21 grid points. This leads to a high enough resolution according to investigations of Ivanell et al. (2008), Wu and Porté-Agel (2012) or Gomes et al. (2014) to avoid any dependence of the wake on the resolution. Generally, the numerical simulation results are plotted in dimensionless coordinates as a function of the rotor diameter $D$. The contour of the actuator in the crosssections represents the transition to a force of zero. Furthermore, only a sector of the complete computational domain is shown in most of the following plots. 


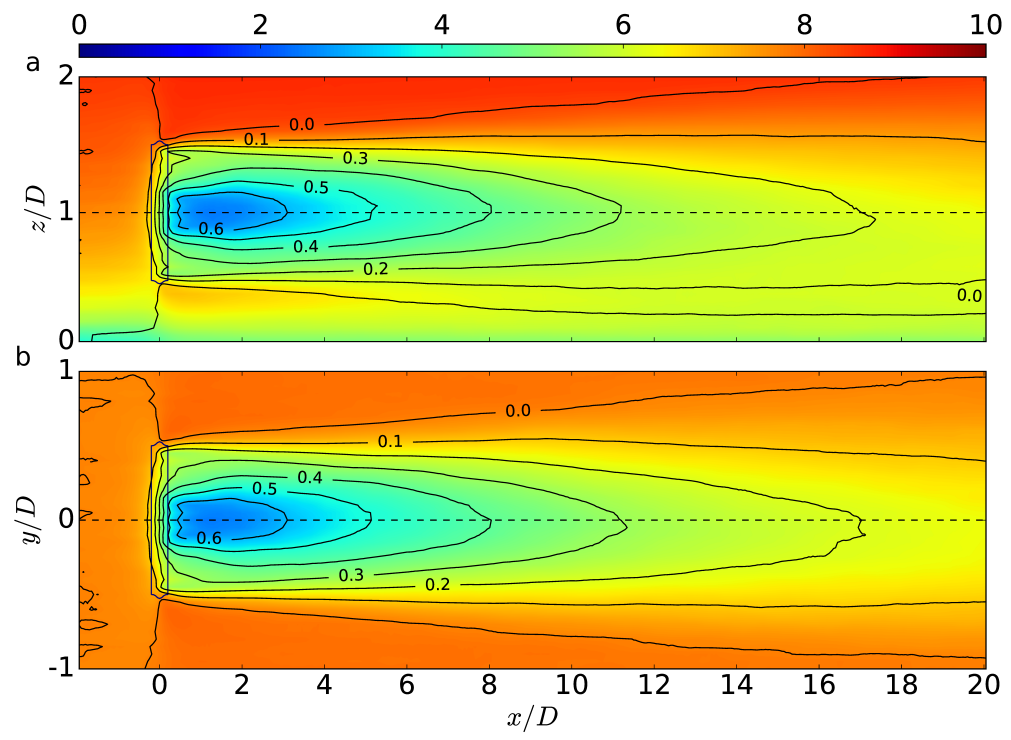

Fig. 2 Streamwise wind field in a vertical $x-z$ cross-section at $y_{0}$ in (a) and in a horizontal $x-y$ cross-section at $z_{h}$ in (b). The contours represent the velocity deficit $\left(u_{\infty, y_{0}, k}-u_{i, y_{0}, k}\right) / u_{\infty, y_{0}, k}$ in (a) and $\left(u_{\infty, j, z_{h}}-u_{i, j, z_{h}}\right) / u_{\infty, j, z_{h}}$ in (b). Note, that in these cross-sections, the scale in the $z$ or $y$-direction is exaggerated compared to the horizontal scale the in $x$-direction.

Now, we investigate the following characteristics of the wake of a wind turbine:

- The spatial distribution of the velocities $u, v$ and $w$.

- The streamwise velocity ratio

$$
V R_{x, y, z}=\frac{\left.<u_{x, y_{0}, z_{h}}\right\rangle_{t}}{\left.<u_{x_{\infty}, y_{0}, z_{h}}\right\rangle_{t}}
$$

as it is related to the power loss of a wind turbine.

- The streamwise turbulent intensity

$$
I_{x, y, z}=\frac{\sigma_{u_{x, y, z}}}{\left.<u_{x, y, z_{h}}\right\rangle_{t}}
$$

with $\sigma_{u_{x, y, z}}=\sqrt{\left\langle u_{x, y, z}^{\prime 2}\right\rangle_{t}}$ and $u_{x, y, z}^{\prime}=u_{x, y, z}-\left\langle u_{x, y, z}>_{t}\right.$, as it affects the flow-induced dynamic loads on downwind turbines.

\subsection{Reference simulation B_1}

Figure 2 shows the vertical (Fig. 2a) and horizontal (Fig. 2b) cross-sections of the streamwise wind field of simulation B_1. The general wake structure reveals 
a minimum of the velocity right behind the rotor with a velocity increase in the radial and streamwise directions. This pattern results from the entrainment of surrounding air with higher velocity values, it is observed prevalently in field experiments in the atmosphere (Heimann et al., 2011, Fig. 3) or in wind-tunnel measurements (Zhang et al., 2012, Fig. 4) as well as simulated numerically (Porté-Agel et al., 2010, Fig. 5; Wu and Porté-Agel, 2012, Fig. 3; Aitken et al., 2014, Fig. 5; Mirocha et al., 2014, Fig. 5).

The $x-y$ cross-section of $u$ shows a nearly axisymmetric distribution (Fig. 2b), whereas the $x-z$ cross-section of $u$ displays a non-axisymmetric mean velocity profile (Fig. 2a) as a consequence of the vertically sheared upstream wind profile and the effect of the surface. Another feature in the $x-z$ cross-section (Fig. 2a) represents the region of higher velocity air at the lowest part of the rotor in comparison to the surroundings. The velocity deficit plotted as contour lines in Fig. 2 enables a comparison with lidar measurements (Iungo et al., 2013; Käsler et al., 2010) or with remotely piloted aircraft measurements (Wildmann et al., 2014). These measurements for similar sized turbines and wind speeds result in a wind speed deficit of about 50 to $60 \%$ at $x=4 D$, which is in line with the contours of the reference simulation in Fig. 2.

In Fig. 3, the mean values of $u, v$ and $w$ are plotted in a $y-z$ cross-sections for selected downstream positions at $x=3 D, x=5 D$ and $x=10 D$. With increasing streamwise distance from the rotor, the flow field $u$ recovers and starts to converge towards the upstream wind profile. The general structure of the position of the velocity minimum as well as the recovery of the wind field is comparable to published results (e.g., Wu and Porté-Agel, 2012, Fig. 4; Mirocha et al., 2014, Fig. 4). Depending on the implementation of a nacelle, the flow field directly behind the centre of the wind turbine changes. Among others, Wu and Porté-Agel (2011) and Meyers and Meneveau (2013) include the nacelle, whereas it is neglected inAitken et al. (2014) and Mirocha et al. (2014). The slices of the lateral wind component $v$ reveal a maximum at the upper rotor part and a minimum at the lower part, which corresponds to the vertical velocity field $w$ with a maximum for $y / D \in[-1,0]$ and a minimum for $y / D \in[0,1]$. The intensity of this rotational effect decreases with increasing streamwise distance from the rotor. The regions with the maximum swirl of the flow are veering away from the rotor centre for an increasing downstream distance. The pattern in $v$ and $w$ is comparable to Mirocha et al. (2014, Fig. 4). In contrast to our results, the $y-z$ cross-sections in Mirocha et al. (2014) are asymmetric, which is most likely induced by the weakly convective ABL in their simulations.

In Fig. 4, the temporally averaged velocity component in streamwise direction $\left\langle u_{x, y, z}\right\rangle_{t}$ is plotted as a function of streamwise distance for different positions (top, bottom, right $(y / D \in[0,1])$, left $(y / D \in[-1,0])) 60$ m away from the centre of the rotor. These positions, although located outside of the actuator $(R=50 \mathrm{~m})$, are still close enough to represent the effect of the forces resulting from Eq. 8 on the flow field. In the upstream region, the velocities at the top and the bottom locations differ due to the incoming logarithmic wind profile whereas the wind speeds right and left of the rotor are the same. 

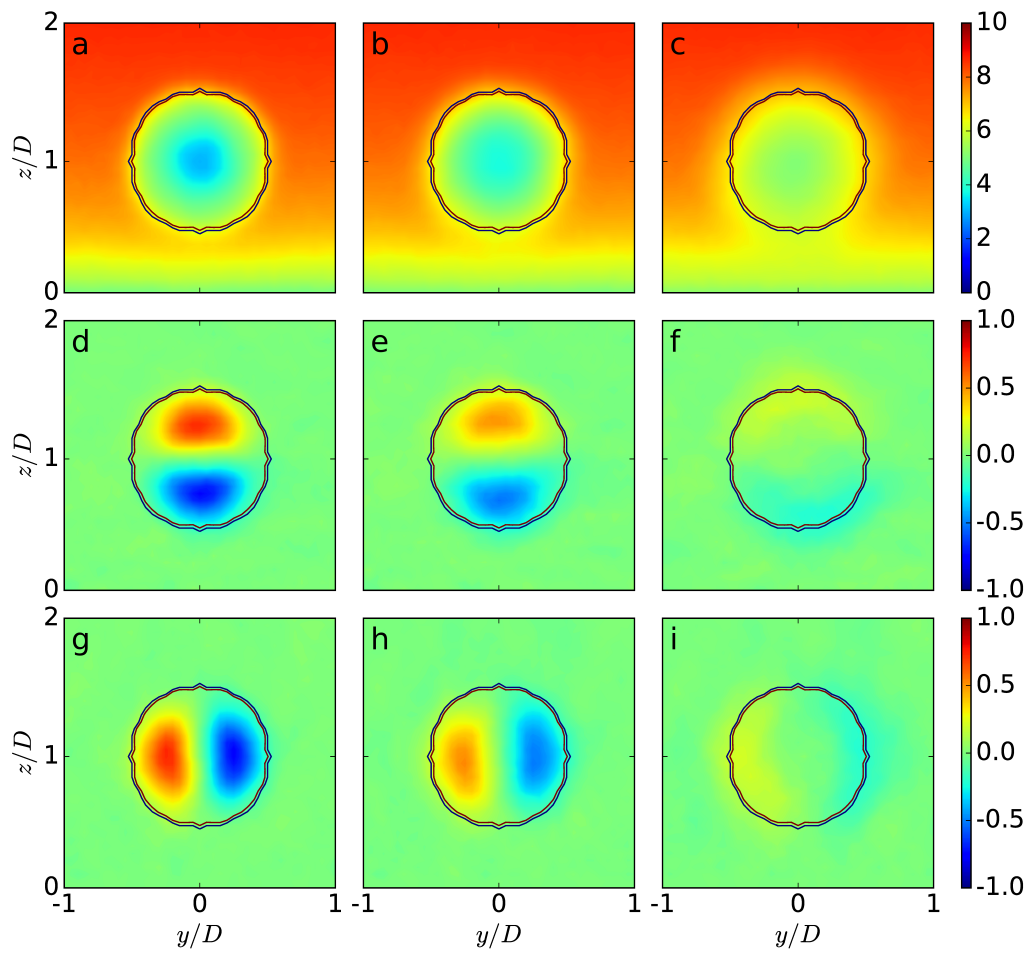

Fig. 3 The averaged values of the base-case simulation (B_1) of $\left\langle u_{x, y, z}\right\rangle_{t}$ in (a)-(c), $\left\langle v_{x, y, z}\right\rangle_{t}$ in (d)-(f) and $\left\langle w_{x, y, z}\right\rangle_{t}$ in (g)-(i) in $y-z$ cross-sections at downstream positions $x=3 D((\mathrm{a}),(\mathrm{d}),(\mathrm{g})), x=5 D((\mathrm{~b}),(\mathrm{e}),(\mathrm{h}))$ and $x=10 D((\mathrm{c}),(\mathrm{f}),(\mathrm{i}))$.

Approaching the rotor, the flow is decelerated in front of the wind turbine and accelerated behind it. This behaviour is induced by the flow deceleration due to the axial force $F_{x}$, which causes a pressure increase in front of the rotor and a decrease behind (Bernoulli equation) (Hansen, 2008). The difference of the flow in the spanwise direction for $x / D>2$ results from the rotation of the actuator, leading to an accelerated (decelerated) flow on the right (left) due to downward (upward) transport of air with higher (lower) momentum. The flow recovers with increasing distance and the velocity values start to approach the values of the incoming wind field for $x \geq 10 D$. The effect of the wind turbine on the wake is not negligible even at a streamwise distance of $x=20 D$ in Fig. 2, therefore we expect a full recovery in Fig. 4 at positions $x>20 D$. 


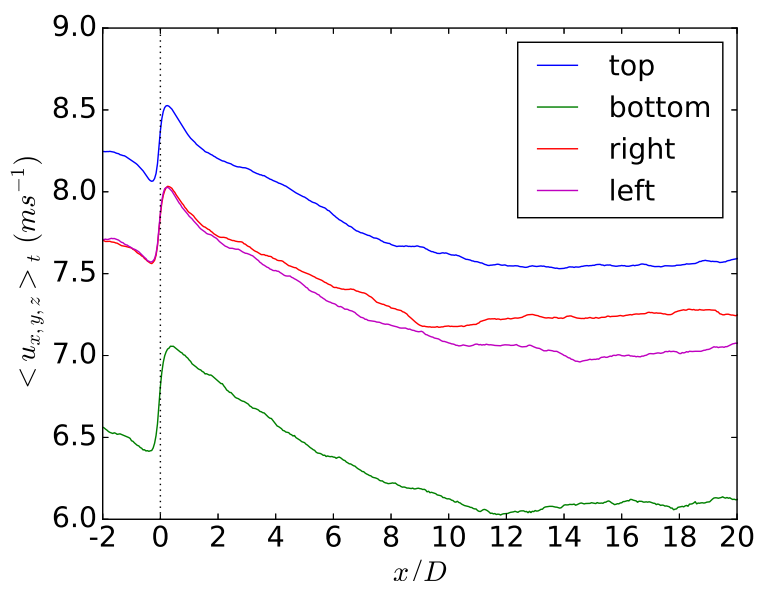

Fig. 4 The velocity component in streamwise direction $\left\langle u_{x, y, z}\right\rangle_{t}$ averaged over the last $t=50 \mathrm{~min}$ of the base-case simulation B_1 at four positions, which are located 60 $\mathrm{m}$ away from the rotor centre $(R=50 \mathrm{~m})$, in both spanwise (left and right) and vertical (top and bottom) directions. The spanwise directions correspond to Figs. 2 and 3 with right $\equiv y / D \in[0,1]$ and left $\equiv y / D \in[-1,0]$.

\subsection{Impact of the perturbation amplitude}

The method of preserving the background turbulence includes the factor $\alpha$ in Eq. 5, which was introduced as the amplitude of the perturbation. The impact of $\alpha$ is studied in simulations B_5 $(\alpha=5)$ and B_10 $(\alpha=10)$ and compared to the reference simulation B_1 $(\alpha=1)$.

Figure 5a shows the streamwise profiles of the velocity ratio from Eq. 16 for different values of the perturbation amplitude $\alpha$. A larger $\alpha$ value leads to a progressively shorter streamwise extension of the wake, induced by a stronger entrainment of ambient air. Further, the minimum of the velocity ratio in the near wake directly behind the nacelle increases.

The markers in Fig. 5a correspond to different wind-turbine studies, as described in detail in the caption of Fig. 5. The simulation results of B_1 are comparable to lidar measurements and WRF-LES model results for a stable ABL (Aitken et al., 2014). By increasing the value of $\alpha$, the velocity ratio approaches values found in observations and simulations of cases with enhanced turbulence. The numerical results of simulation B_5 correspond to a neutral ABL (Wu and Porté-Agel, 2011; Gomes et al., 2014), whereas the results of simulation B_10 are almost comparable to measurements and WRF-LES model results in a convective ABL (Mirocha et al., 2014). This comparison with other studies leads to the hypothesis that the factor $\alpha$ from Eq. 5 could be related quantitatively to different levels of atmospheric turbulence.

We also tested various precursor simulations (convection or Coriolis force as trigger to excite turbulence) resulting in different spectral energy densities. The velocity ratio for a larger amount of the spectral energy density is in 


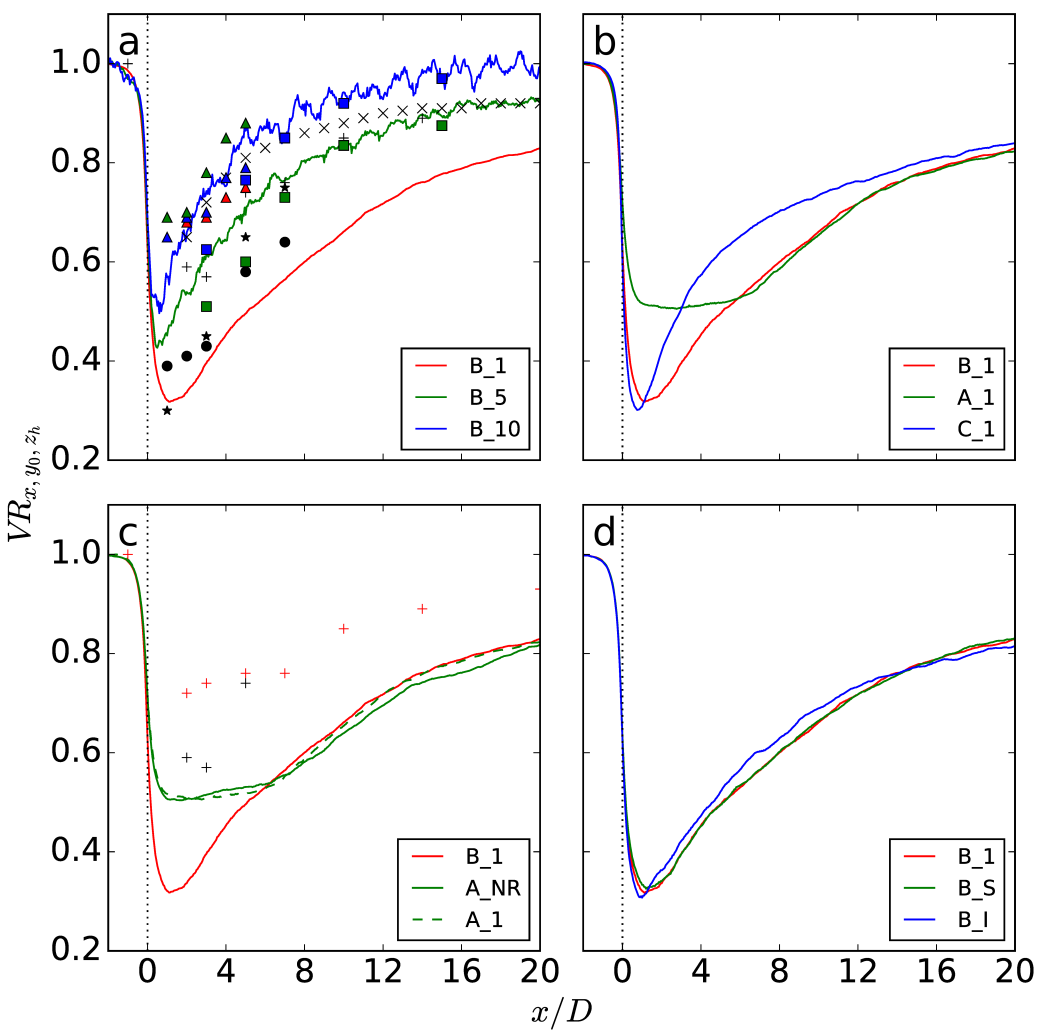

Fig. 5 The streamwise dependence of the velocity ratio from Eq. 16 at $y_{0}$ and $z_{h}$ for all simulations listed in Table 4, grouped together regarding the wake impact of the perturbation amplitude in (a), the wind-turbine parametrization in (b), the rotation of the disc in (c), and the SGS closure model in (d). The markers in (a) and (c) correspond to the results of the velocity ratio from the wake of a wind turbine in various studies: the values marked by a plus sign are extracted out of the LES from Wu and Porté-Agel (2011, Fig. 4) for a neutral ABL. The crosses correspond to the neutral ABL RANS simulation by Gomes et al. (2014, Fig. 1). The circles are extracted from lidar measurements in a stable ABL and the asterisks from the corresponding WRF-LES model simulation of a stable ABL, see Aitken et al. (2014, Fig. 6). The red triangles are extracted from convective ABL measurements, the blue triangles correspond to the WRF-LES model simulation of a convective ABL characterized by a heat flux of $20 \mathrm{~W} \mathrm{~m}^{-2}$, and the green triangles correspond to the WRF-LES model simulation of a convective ABL characterized by a heat flux of $100 \mathrm{~W} \mathrm{~m}^{-2}$, investigated in Mirocha et al. (2014, Fig. 8). The green squares correspond to a neutral ABL with a roughness length $z_{0}=1 \times 10^{-5} \mathrm{~m}$, and the blue squares to a value of $z_{0}=1 \times 10^{-1} \mathrm{~m} \mathrm{(Wu}$ and Porté-Agel, 2012, Fig. 5). The red plus signs in (c) correspond to the results of the non-rotating disc in Wu and Porté-Agel (2011, Fig. 4) opposed to their rotating results in black. 


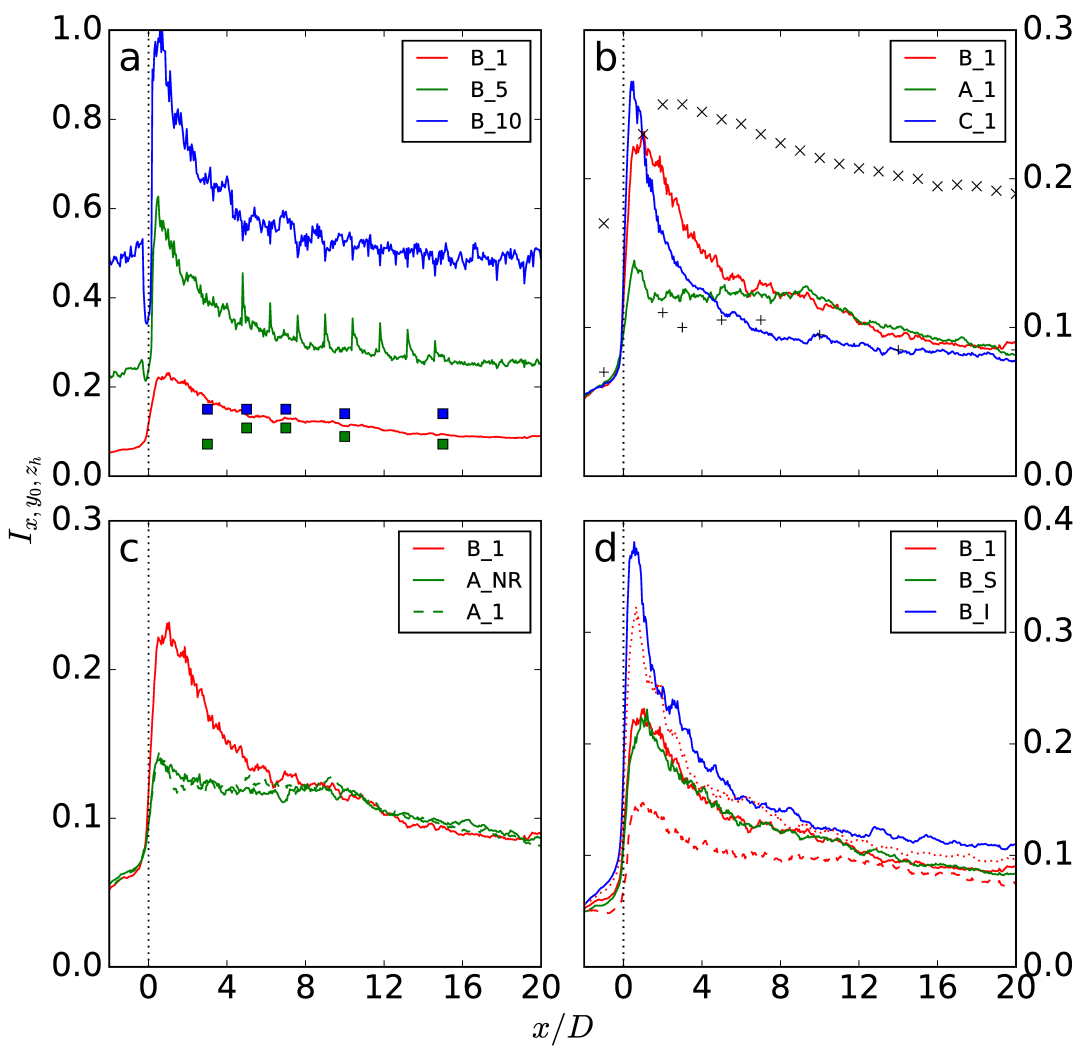

Fig. 6 The streamwise dependence of the turbulence intensity from Eq. 17 at $y_{0}$ and $z_{h}$ for all simulations listed in Table 4, grouped together regarding the wake impact of the perturbation amplitude in (a), the wind-turbine parametrization in (b), the rotation of the disc in (c), and the SGS closure model in (d). The markers in (a) and (b) result from the streamwise turbulent intensity in the wake of a wind turbine in various studies: the green squares in (a) correspond to a neutral ABL with a roughness length $z_{0}=1 \times 10^{-5} \mathrm{~m}$, and the blue squares to a value of $z_{0}=1 \times 10^{-1} \mathrm{~m}$ (Wu and Porté-Agel, 2012, Fig. 8). The values marked by a plus sign in (b) are extracted out of the LES from Wu and Porté-Agel (2011, Fig. 7) for a neutral ABL. The crosses correspond to the neutral ABL RANS simulation by Gomes et al. (2014, Fig. 1). The dotted line in plot (d) represents simulation B_1 with $1 / 2$ times the length scale in the SGS closure model, whereas the dashed line represents simulation B_1 with twice the length scale in the SGS closure model.

agreement with a larger value of $\alpha$ (not shown here). The parameter $\alpha$ is also comparable to the different roughness lengths used in Wu and Porté-Agel (2012), with a larger roughness length corresponding to a higher perturbation amplitude.

The streamwise profiles of the turbulent intensity in Eq. 17 are presented in Fig. 6a for different $\alpha$ values. The turbulent intensity $I_{x, y_{0}, z_{h}}$ increases 
with increasing $\alpha$. In the upstream as well as in the downstream region, the streamwise distribution of $I_{x, y_{0}, z_{h}}$ is proportional to $\alpha$. Wu and Porté-Agel (2012) investigate an increase of $\mathrm{I}_{x, y_{0}, z_{h}}$ for increasing $z_{0}$. We also result in an increase of $\mathrm{I}_{x, y_{0}, z_{h}}$ for increasing $\alpha$, reinforcing our assumption that larger $\alpha$ values are comparable to a surface with an increased roughness length.

We conclude that the entrainment in the wake can be easily modified by adjusting the value of $\alpha$ in the numerical simulations. In this way, a realistic level of atmospheric background turbulence intensity corresponding to various atmospheric stratifications or different roughness lengths can be parametrized by applying our turbulence preserving model.

\subsection{Impact of the wind-turbine parametrization}

The impact of the three wind-turbine parametrizations $\mathrm{A}, \mathrm{B}$, and $\mathrm{C}$ on the wake is studied for $\alpha=1$ in simulations A_1, B_1 and C_1. The different parametrizations influence the velocity ratio in the wake as documented in Fig. $5 \mathrm{~b}$.

A comparison between simulation A_1 and simulation B_1 focuses on the difference between the MMT and the BEM method. Approaching a downstream distance of $x=5 D$, the difference in the wake structure becomes marginal. Therefore, we define a streamwise distance of $x=5 D$ as the transition between the near wake and the far wake. Further, the value of the minimum of the velocity ratio in the near wake is larger for parametrization A in A_1 due to no radial dependence of the thrust and power coefficients in Eqs. 6 and 7 .

The difference between parametrizations $\mathrm{B}$ and $\mathrm{C}$ are the local blade characteristics of the two airfoils. In parametrization $\mathrm{C}$ the velocity field in the streamwise direction recovers more rapidly up to approximately $x=14 D$ in comparison to type B. This is caused by the sharper gradient in the axial force at the edge of the nacelle between $0.2 r / R$ and $0.3 r / R$ in Fig. 1 .

The different parametrizations also have an impact on the value of the maximum of the turbulent intensity in Fig. 6b. The maximum is larger for parametrization B in comparison to parametrization $\mathrm{A}$. This is caused by the radial gradient of the axial force in parametrization $\mathrm{B}$, which contrasts a constant force in parametrization A, as shown in Fig. 1. The streamwise turbulent intensities of parametrizations A and B are very similar in the far wake. The difference in the maximum between parametrizations $\mathrm{B}$ and $\mathrm{C}$ correlates with the gradient of the axial force close to the nacelle in Fig. 1. A larger maximum corresponds to a sharper gradient. A sharper gradient also results in a more rapid decline in parametrization $\mathrm{C}$ in comparison to parametrization $\mathrm{B}$ up to approximately $x=14 D$.

Comparing these results to other studies, the turbulent intensity values of all three parametrizations are rather small in comparison to the RANS simulation of Gomes et al. (2014) approaching $x \geq 2 D$. A comparison with the LES of Wu and Porté-Agel (2011) results in a rather good agreement in the 
near wake for parametrization A and in the far wake for parametrization C. The agreement of parametrization $\mathrm{C}$ is referable to a similar radial distribution of the forces yielded from the same blade characteristics.

We conclude that the MMT is sufficient as simplification of the BEM parametrization if only the far wake is of interest. In the near wake the radial dependence of the axial force becomes important. Further, the local blade characteristics influence the wake up to a downstream distance of $x=14 D$.

In the scope of this work, we also implemented an advanced version of the MMT. It considers the radial distribution of the forces in Eqs. 6 and 7, which is adopted from the radial chord length dispersion in Micallef et al. (2013). The forces in Eqs. 6 and 7 are modified similarly to the procedure in Gomes et al. (2014). Numerical simulations using this approach led to a better agreement of the near-wake structure with the BEM method in parametrization B in comparison to the MMT approach (not shown here).

\subsection{Impact of the rotation of the disc}

To investigate the impact of the rotation of the actuator on the wake structure, simulation A_NR with parametrization A, no rotation of the disc $\left(F_{\Theta}=0\right.$ in Eq. 7) and $\alpha=1$ is performed and compared to simulations A_1 and B_1.

The minimum of the velocity ratio in simulation B_1 is smaller in comparison to simulation A_NR. This finding is in agreement with the results of $\mathrm{Wu}$ and Porté-Agel (2011) (markers in Fig. 5c). A comparison between simulation A_1 and simulation A_NR results in a marginal impact of the tangential force on the streamwise velocity ratio according to Fig. $5 \mathrm{c}$. Therefore, the difference between simulation B_1 and simulation A_NR is evoked by the uniform thrust force distribution over the disc, which has a larger impact on the velocity ratio than the marginal effect of rotation.

Wu and Porté-Agel (2011) show an increase of the turbulence intensity applying the BEM method instead of the classical Rankine-Froude approach. The streamwise turbulent intensity at the centre line in Fig. $6 \mathrm{c}$ is also larger for the BEM parametrization in the near wake. The effect of rotation is marginal. Consequently, not the swirl, but the non-uniform distribution of the axial force in the BEM method (Fig. 1) is responsible for the near-wake difference in the streamwise turbulent intensity in Fig. 6c.

The rotation of the disc in simulation A_1 leads to a swirl in the wake as shown in Figs. 7a-c. The rotational effect of the disc is evident at $x=3 D$. Approaching $x=10 D$, the swirl in the disc region decays while it is transported outwards. Both effects originate from entrainment processes. At a downstream position of $x=20 D$, the rotation in the disc region approaches zero, whereas there is still some swirl in the air around the disc. In contrast to this rotational behaviour, there is no swirl of the air downstream of the non-rotating disc of simulation A_NR in Figs. 7d-f. The pattern of the streamwise velocity $u$ in the rotor region as well as in the surroundings are comparable in both simulations at $x=3 \mathrm{D}$ and $10 \mathrm{D}$. At $x=20 \mathrm{D}$, the wake pattern in simulation A_NR 


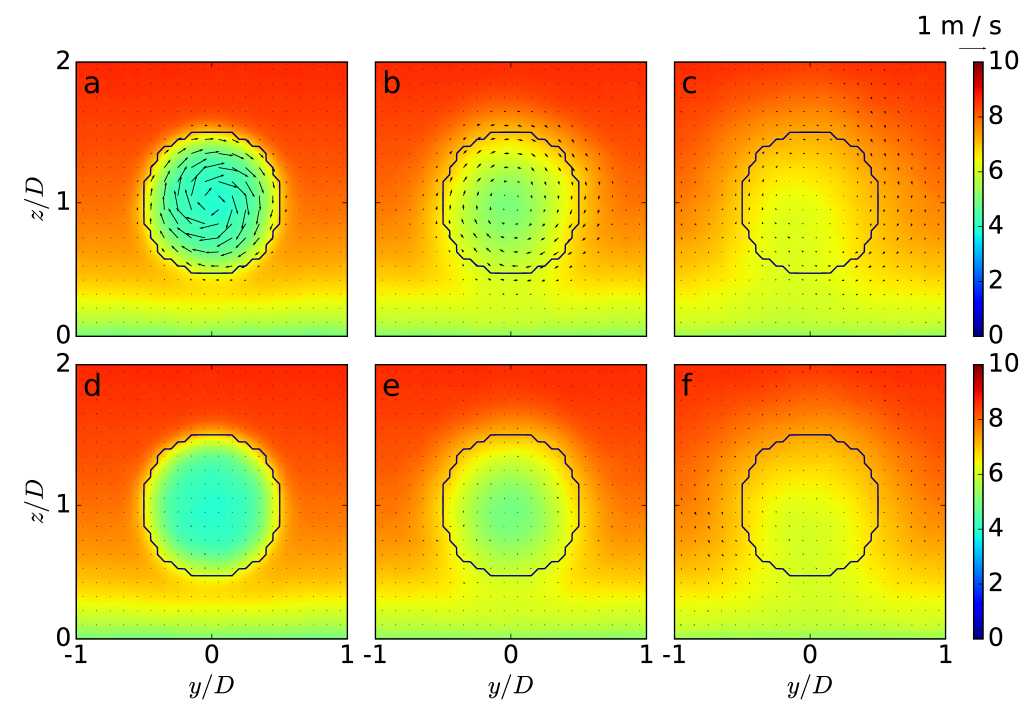

Fig. 7 The averaged value of $\left\langle u_{x, y, z}\right\rangle_{t}$ in a $y$ - $z$ cross-section at downstream positions $x=3 D((\mathrm{a}),(\mathrm{d})), x=10 D((\mathrm{~b}),(\mathrm{e}))$ and $x=20 D((\mathrm{c}),(\mathrm{f}))$ for simulation A_1 ((a)(c)) and simulation A_NR ((d)-(f)). The arrows represent the wind vectors $\left(\left\langle v_{x, y, z}\right\rangle_{t}\right.$, $\left.<w_{x, y, z}>_{t}\right)$. The magnitude of $1 \mathrm{~m} \mathrm{~s}^{-1}$ is shown at the right edge of the plot.

is symmetric, whereas in simulation A_1 it is shifted towards $y / D \in[-1,0]$. This asymmetric streamwise velocity field results from the rotation of the disc and is also prevalent in the study of Wu and Porté-Agel (2012, Fig. 4).

This investigation leads to the conclusion that the rotation has a minor effect on the velocity ratio and on the streamwise turbulent intensity at the centre line. However, the effect of the tangential force on the $v$ and $w$ wind components is prevailing even in the far-wake region, with an influence on the streamwise velocity field in the $y$ - $z$ plane.

\subsection{Impact of the SGS closure model}

The impact of the SGS closure models is investigated by comparing the TKE SGS closure model simulation B_1 with the Smagorinsky SGS closure model simulation B_S. The geophysical flow solver EULAG provides a reliable numerical testbed to study the SGS closure model sensitivities. Further, it depends on the NFT integrations of Eqs. 1 to 3 and therefore offers the possibility to integrate these equations without an explicit SGS closure model by setting $\mathcal{V}=0$ and $\mathcal{H}=0$ in Eqs. 1 and 2 in the implicit LES B_I.

The streamwise dependence of the velocity ratios in Fig. 5d agrees quantitatively very well for simulation B_1 and simulation B_S. The contrast to simulation B_I is insignificant. 
The turbulent intensities in Fig. 6d are also rather similar for the TKE and 664 the Smagorinsky SGS closure model. For the implicit LES, the maximum of ${ }_{665} I_{x, y_{0}, z_{h}}$ is roughly 1.7 times larger than in the simulations with the SGS closure model. In the far wake the difference becomes rather small. The dependency of the difference in the turbulent intensity in the near wake between an implicit LES and a simulation using an explicit SGS closure model is verified with two further simulations, modifying the SGS closure model of simulation B_1. In the first simulation, the length scale of the TKE SGS closure model is multiplied by a factor of $1 / 2$, resulting in the dotted red line in Fig. $6 \mathrm{~d}$, whereas in the second simulation, the length scale is multiplied by a factor of 2 , resulting in the dashed red line. Decreasing (increasing) the length scale of the closure model results in a weaker (stronger) damping. A weaker damping induces larger turbulence, approaching the turbulent intensity behaviour of the implicit LES, whereas a stronger damping results in a weaker turbulent behaviour. The streamwise velocity ratios are nearly unaffected by the length scale of the closure model (not shown here).

The agreement between the established SGS schemes (TKE and Smagorinsky) is a remarkable result and confirms earlier findings by Smolarkiewicz et al. (2007). The possibility of an implicit LES of wind-turbine flows enables numerical simulations with stretched or adaptive meshes, where an explicit SGS parametrization might be difficult and troublesome.

The length scale of the closure model offers another tuning parameter in addition to $\alpha$, which can explain the difference in the streamwise turbulent intensity in comparison to other simulation results of Wu and Porté-Agel (2011), Wu and Porté-Agel (2012) and Gomes et al. (2014).

\section{Conclusion}

The wake characteristics of a wind turbine in a turbulent and neutral ABL flow were investigated by means of LES. Besides reliable wind-turbine parametrizations, an effective method to preserve the atmospheric background turbulence was applied successfully in the numerical solver. The numerical simulations using these two ingredients result in realistic wake structures, which are quantitatively comparable with previous observations and numerical simulation results.

The atmospheric background turbulence field was simulated by a precursor simulation of the neutral ABL using cyclic boundary conditions. Velocity perturbations were extracted once from the equilibrium state of the precursor simulation. These perturbation velocities were superimposed on the flow field of the wind-turbine simulations by a new method suitable for open horizontal boundaries. This method preserves the atmospheric background turbulence by applying the spectral energy distribution at every timestep taken from three $3 \mathrm{D}$ fields $(u, v, w)$ of the precursor simulation. The newly developed turbulence preserving method uses an empirical factor $\alpha$, which controls the energy content of the background turbulence. Larger $\alpha$ values refer to more turbulent 
flow regimes, e.g. under convective conditions or for flows over a surface with an increased roughness length. An increase of the atmospheric background turbulence, i.e. larger $\alpha$ values, enhance the entrainment of air into the wake, resulting in a shorter streamwise wake extension and an increase of the streamwise turbulent intensity. The turbulence preserving method as presented here provides a simple and numerically very effective tool for studying the interaction of ABL flow of different thermal stratifications with a wind turbine by applying the same spectral energy distribution and varying the parameter $\alpha$. Considering different stratifications of the atmosphere is important, as a near-neutral stratification occurs only with a frequency of roughly $10 \%$ according to data from a field experiment (SWiFT Facility Representation and Preparedness; 730 days of measurement in the period from 2012 to 2014 (Sue Ellen Haupt (NCAR), personal communication, 2015)).

Furthermore, the wake structure was investigated for different wind-turbine parametrizations. We considered the MMT and the BEM method as windturbine parametrizations, varied the local blade characteristics in the BEM method and studied the effect of rotation of the actuator. The BEM method yields a more accurate prediction of the near-wake characteristics if the airfoil data of the wind turbine are known. Considering how sparse information on detailed blade geometries is available, the MMT offers an alternative. It was found that the MMT is a reasonable simplification of the BEM model for studies of the far wake, when near-wake characteristics are of secondary importance. The wake structure for the two considered airfoils in the BEM model differs up to a streamwise distance of $14 D$. The very far wake is not affected by the blade characteristics. The rotation of the wind turbine leads to a swirl in the wake and impacts on the streamwise velocity field in the $y-z$ plane even in the far wake.

The sensitivity of the wake to two SGS closure models (TKE and Smagorinsky-type models) and numerical simulations without an explicit SGS closure model (implicit LES) was studied. The choice of the SGS closure models has a rather small impact on the wake characteristics. Even the implicit LES results of the streamwise velocity ratio agree surprisingly well with the former simulations reinforcing the suitability of this approach to study a wide class of ABL flows. However, there is a remarkable impact on the streamwise turbulent intensity in the near wake, which is strongly affected by the amount of damping in the SGS closure model.

In this study, we presented a simple and numerically effective method to perform LES of wind turbines with a realistic background turbulence field. Our turbulence preserving model as well as the wind-turbine models, both implemented in the numerical model EULAG, allow for subsequent future applications for a wide range of scales, for different thermal stratifications, as well as for flows over heterogeneous and hilly terrains. 
project, funded by the Federal Ministry for the Environment, Nature Conservation, Building and Nuclear Safety by a resolution of the German Federal Parliament. The authors gratefully acknowledge the Gauss Centre for Supercomputing e.V. (www.gauss-centre.eu) for funding this project by providing computing time on the GCS Supercomputer SuperMUC at Leibniz Supercomputing Centre (LRZ, www.lrz.de). 
Appendix: BEM parameters

Table 5 List of the BEM method parameters used in parametrization of type B (10 MW reference wind turbine from DTU) (Mark Zagar (Vestas), personal communication, 2015) and type C (three-blade GWS/EP-6030x3 rotor) (Wu and Porté-Agel, 2011). The radius $r$ and the chord length $c$ of the two rotors are scaled to a rotor diameter of $100 \mathrm{~m}$.

\begin{tabular}{c|c|c|c|c|c}
\multicolumn{3}{|c|}{ parametrization B } & \multicolumn{3}{|c}{ parametrization $\mathrm{C}$} \\
$r / \mathrm{m}$ & $c(r) / \mathrm{m}$ & $\Theta(r) /{ }^{\circ}$ & $r / \mathrm{m}$ & $c(r) / \mathrm{m}$ & $\Theta(r) /{ }^{\circ}$ \\
\hline 5.0 & 5.3 & 13.3 & 6.7 & 9.3 & 20.5 \\
\hline 10.0 & 6.0 & 13.2 & 13.3 & 9.8 & 20.9 \\
\hline 15.0 & 6.2 & 10.5 & 20.0 & 9.8 & 19.8 \\
\hline 20.0 & 5.8 & 9.0 & 26.6 & 9.4 & 16.9 \\
\hline 25.0 & 5.0 & 7.3 & 33.3 & 8.7 & 13.2 \\
\hline 30.0 & 4.5 & 5.5 & 40.0 & 7.9 & 10.7 \\
\hline 35.0 & 3.5 & 3.8 & 46.7 & 6.8 & 9.1 \\
\hline 40.0 & 3.0 & 2.5 & 50.0 & 4.0 & 6.7 \\
\hline 45.0 & 2.3 & 1.3 & & & \\
\hline 50.0 & 1.0 & 0.2 & & & \\
\hline
\end{tabular}




\section{References}

Aitken ML, Kosović B, Mirocha JD, Lundquist JK (2014) Large eddy simulation of wind turbine wake dynamics in the stable boundary layer using the Weather Research and Forecasting Model. J Renew Sust Energy 6:1529 1539

Bellon G, Stevens B (2012) Using the sensitivity of large-eddy simulations to evaluate atmospheric boundary layer models. J Atmos Sci 69:1582-1601

Betz A (1926) Windenergie und ihre Ausnutzung in Windmühlen

Calaf M, Meneveau C, Meyers J (2010) Large eddy simulation study of fully developed wind-turbine array boundary layers. Phys Fluids 22:015110

Chamorro LP, Porté-Agel F (2009) A wind-tunnel investigation of windturbine wakes: Boundary-layer turbulence effects. Boundary-Layer Meteorol 132:129-149

Doyle JD, Gaberšek S, Jiang Q, Bernardet L, Brown JM, Dörnbrack A, Filaus E, Grubišic V, Kirshbaum DJ, Knoth O, et al. (2011) An intercomparison of t-rex mountain-wave simulations and implications for mesoscale predictability. Mon Weather Rev 139:2811-2831

El Kasmi A, Masson C (2008) An extended model for turbulent flow through horizontal-axis wind turbines. J Wind Eng Ind Aerodyn 96:103-122

Emeis S (2013) Wind energy meteorology: Atmospheric physics for wind power generation. Springer Science \& Business Media, 196 pp

Emeis S (2014) Current issues in wind energy meteorology. Meteorol Appl 21:803-819

Fröhlich J (2006) Large Eddy Simulation turbulenter Strömungen. Teubner Verlag / GWV Fachverlage GmbH, Wiesbaden, 414 pp

Froude RE (1889) On the part played in propulsion by difference of fluid pressure. Trans RINA 30:390

Glauert H (1963) Airplane propellers. In: Aerodynamic theory, W. F. Durand, Dover, New York, pp 169-360.

Gomes VMMGC, Palma JMLM, Lopes AS (2014) Improving actuator disk wake model. In: The science of making torque from wind. Conference series, vol 524, p 012170.

Grinstein FF, Margolin LG, Rider WJ (2007) Implicit Large Eddy Simulation. Cambridge university press, $546 \mathrm{pp}$

Hansen MO (2008) Aerodynamics of wind turbines, vol 2. Earthscan, London and Sterling, UK and USA, $181 \mathrm{pp}$

Heimann D, Käsler Y, Gross G (2011) The wake of a wind turbine and its influence on sound propagation. Meteorol Z 20:449-460

Iungo GV, Wu YT, Porté-Agel F (2013) Field measurements of wind turbine wakes with lidars. J Atmos Ocean Technol 30:274-287

Ivanell BS, Mikkelsen R, Henningson D (2008) Validation of methods using EllipSys3D. Technical report, KTH, TRITA-MEK 2008:12, pp 183-221.

Käsler Y, Rahm S, Simmet R, Kühn M (2010) Wake measurements of a multiMW wind turbine with coherent long-range pulsed doppler wind lidar. J Atmos Ocean Technol 27:1529-1532 
Kataoka H, Mizuno M (2002) Numerical flow computation around aeroelastic 3D square cylinder using inflow turbulence. Wind and Structures 5:379-392

Kühnlein C, Smolarkiewicz PK, Dörnbrack A (2012) Modelling atmospheric flows with adaptive moving meshes. J Comput Phys 231:2741-2763

Mann J (1994) The spatial structure of neutral atmospheric surface-layer turbulence. J Fluid Mech 273:141-168

Manwell J, McGowan J, Roger A (2002) Wind Energy Explained: Theory, Design and Application. Wiley: New York, NY, USA, 134 pp

Margolin L, Rider W (2002) A rationale for implicit turbulence modelling. International Journal for Numerical Methods in Fluids 39:821-841

Margolin L, Smolarkiewicz PK, Wyszogrodzki A (2002) Implicit turbulence modeling for high reynolds number flows. Journal of Fluids Engineering 124:862-867

Margolin L, Rider W, Grinstein F (2006) Modeling turbulent flow with implicit les. Journal of Turbulence 7:1-27

Medici D, Alfredsson PH (2006) Measurements on a wind turbine wake: 3D effects and bluff body vortex shedding. Wind Energy 9:219-236

Meyers J, Meneveau C (2013) Flow visualization using momentum and energy transport tubes and applications to turbulent flow in wind farms. J Fluid Mech 715:335-358

Micallef D, Bussel GV, Sant T (2013) An investigation of radial velocities for a horizontal axis wind turbine in axial and yawed flows. Wind Energy $16: 529-544$

Mikkelsen R (2003) Actuator disc methods applied to wind turbines. PhD thesis, Technical University of Denmark

Mirocha J, Kirkil G, Bou-Zeid E, Chow FK, Kosović B (2013) Transition and equilibration of neutral atmospheric boundary layer flow in one-way nested large-eddy simulations using the weather research and forecasting model. Mon Weather Rev 141:918-940

Mirocha J, Kosovic B, Aitken M, Lundquist J (2014) Implementation of a generalized actuator disk wind turbine model into the weather research and forecasting model for large-eddy simulation applications. J Renew Sust Energy 6:013104

Muñoz-Esparza D, Kosović B, Mirocha J, van Beeck J (2014) Bridging the transition from mesoscale to microscale turbulence in numerical weather prediction models. Boundary-Layer Meteorol 153:409-440

Naughton JW, Heinz S, Balas M, Kelly R, Gopalan H, Lindberg W, Gundling C, Rai R, Sitaraman J, Singh M (2011) Turbulence and the isolated wind turbine. In: 6th AIAA Theoretical Fluid Mechanics Conference, Honolulu, Hawaii, pp 1-19.

Porté-Agel F, Lu H, Wu YT (2010) A large-eddy simulation framework for wind energy applications. In: The Fifth International Symposium on Computational Wind Engineering, vol 23-27 May 2010, Chapel Hill, North Carolina, USA

Prusa JM, Smolarkiewicz PK, Wyszogrodzki AA (2008) EULAG, a computational model for multiscale flows. Computers \& Fluids 37:1193-1207 
Rankine WJM (1865) On the mechanical principles of the action of propellers. Trans RINA 6:13

Schetz JA, Fuhs AE (1996) Handbook of fluid dynamics and fluid machinery. Wiley, New York, $2776 \mathrm{pp}$

Smolarkiewicz PK, Charbonneau P (2013) EULAG, a computational model for multiscale flows: An MHD extension. J Comput Phys 236:608-623

Smolarkiewicz PK, Dörnbrack A (2008) Conservative integrals of adiabatic durran's equations. Int J Numer Methods Fluids 56:1513-1519

Smolarkiewicz PK, Margolin LG (1993) On forward-in-time differencing for fluids: extension to a curviliniear framework. Mon Weather Rev 121:18471859

Smolarkiewicz PK, Margolin LG (1998) MPDATA: A Finite-Difference Solver for Geophysical Flows. J Comput Phys 140:459-480

Smolarkiewicz PK, Prusa JM (2002) Forward-in-time differencing for fluids: simulation of geophysical turbulence. In: Turbulent Flow Computation, Kluwer Academic Publishers, Boston, USA, pp 279-312

Smolarkiewicz PK, Prusa JM (2005) Towards mesh adaptivity for geophysical turbulence: continuous mapping approach. Int J Numer Methods Fluids 47:789-801

Smolarkiewicz PK, Pudykiewicz JA (1992) A class of semi-Lagrangian approximations for fluids. J Atmos Sci 49:2082-2096

Smolarkiewicz PK, Winter CL (2010) Pores resolving simulation of darcy flows. J Comput Phys 229:3121-3133

Smolarkiewicz PK, Sharman R, Weil J, Perry SG, Heist D, Bowker G (2007) Building resolving large-eddy simulations and comparison with wind tunnel experiments. J Comput Phys 227:633-653

Tossas LAM, Leonardi S (2013) Wind turbine modeling for computational fluid dynamics: December 2010-December 2012. NREL Technical Monitor: Pat Moriarty, pp 1-48.

Troldborg N, Sørensen JN, Mikkelsen R (2007) Actuator line simulation of wake of wind turbine operating in turbulent inflow. In: The science of making torque from wind. Conference series, vol 75, p 012063.

Wedi NP, Smolarkiewicz PK (2004) Extending gal-chen and somerville terrainfollowing coordinate transformation on time-dependent curvilinear boundaries. J Comput Phys 193:1-20

Wedi NP, Smolarkiewicz PK (2006) Direct numerical simulation of the plumbMcEwan laboratory analog of the QBO. J Atmos Sci 63:3226-3252

Wildmann N, Hofsäß M, Weimer F, Joos A, Bange J (2014) MASC-a small remotely piloted aircraft (RPA) for wind energy research. Advances in Science and Research 11:55-61

Witha B, Steinfeld G, Heinemann D (2014) High-resolution offshore wake simulations with the LES model PALM. In: Wind energy - Impact of turbulence, Spring 2012, Oldenburg, Germany, pp 175-181.

Wu YT, Porté-Agel F (2011) Large-Eddy Simulation of Wind-Turbine Wakes: Evaluation of Turbine Parametrisations. Boundary-Layer Meteorol 138:345366 
Wu YT, Porté-Agel F (2012) Atmospheric turbulence effects on wind-turbine wakes: An LES study. Energies 5:5340-5362

Zhang W, Markfort CD, Porté-Agel F (2012) Near-wake flow structure downwind of a wind turbine in a turbulent boundary layer. Exp Fluids 52:1219 1235 